\section{Prophylaxe des Schlaganfalls}

\author{
M. Endres ${ }^{1,2}$ \\ 1 Klinik und Hochschulambulanz für Neurologie \\ ${ }^{2}$ Centrum für Schlaganfallforschung Berlin, \\ Charité Universitätsmedizin Berlin
}

In Deutschland ereignen sich pro Jahr etwa 270000 Schlaganfälle, davon $>90 \%$ ischämische Hirninfarkte und $<10 \%$ intrazerebrale Blutungen. Allein in der Stadt Berlin treten > 14000 Schlaganfälle auf, d.h. 1-2 pro Stunde. Jeder dritte Schlaganfall tritt bei einem Patienten auf, der bereits einmal einen Schlaganfall erlitten hat. Die Schlaganfall-Patienten stellen also für die Prophylaxe ein besonderes Kollektiv dar, dessen Risiko im Rahmen der Sekundärprävention bestmöglich reduziert werden sollte.

Die klassischen Säulen der Sekundärprävention stellen dar:

1. Blutdrucksenkung,

2. Cholesterinsenkung mit Statinen (HMG-CoA-Reduktasehemmern) sowie

3. Behandlung mit Thrombozytenfunktionshemmern.

Diese drei medikamentösen Therapien können bei dem Gros der Schlaganfall-Patienten mit arteriothrombotischer Genese angewandt werden.

4. Ein weiteres zentrales Therapieprinzip stellt die Antikoagulation bei kardialer Embolie, insbesondere bei Patienten mit nicht-rheumatischem Vorhofflimmern, dar.

5.Zuletzt müssen Patienten mit symptomatischer Karotisstenose, d.h. Patienten mit hochgradiger Stenose und gerade zurückliegendem Hirninfarkt im ipsilateralen Strombahngebiet durch eine Operation (Thrombendarteriektomie) oder ggf. einen interventionellen Eingriff (Stent-Angioplastie) versorgt werden.

Sicherlich ist es ein besonderes Anliegen der klinischen wie der Grundlagenforschung, neue Therapieprinzipien oder ggf. neue Substanzen zu entwickeln, welche dann in klinischen Studien überprüft und erstmals bei Patienten angewandt werden können (sogenannte Typ-I-Translation). Entscheidend für die bevölkerungsweite Risikoreduktion ist jedoch vielmehr die breite Anwendung und Umsetzung der bereits jetzt etablierten Standardtherapien in der Praxis (Typ-IITranslation). So könnte beispielsweise durch konsequente Blutdruckbehandlung in Deutschland die Zahl der Schlaganfälle halbiert werden. Ebenso stellt das Vorhofflimmern, welches schätzungsweise eine Mio. Patienten in Deutschland betrifft, eine leicht behandelbare und hochrelevante Ursache für Schlaganfälle in Deutschland dar. Insbesondere ist jedoch bei Hochrisiko-Patienten, gerade bei den Schlaganfall-Patienten, die Medikamenten-Adhärenz ein großes Problem. So konnte beispielsweise eine groß angelegte Registerstudie in Schweden zeigen, dass die Versorgung mit den klassischen Schlaganfall-Medikamenten (Plättchenhemmer, Blutdruckmedikamente, Statine) nach wenigen Monaten deutlich abgesunken war. Insbesondere die hochrelevante Antikoagulation bei Schlaganfall-Patienten mit Vorhofflimmern sank auf deutlich $<50 \%$. Innovative Ansätze für die Verbesserung der Medikamenten-Adhärenz wie z.B. über die Ausgabe von Medikamentenblistern über die Apotheken stellen hochwillkommene wissenschaftliche Ansätze dar: Medikamente wirken nicht bei Patienten, die sie nicht nehmen!

In vielen Bereichen der Schlaganfall-Prävention haben sich in den letzten Jahren behandlungsrelevante Neuerungen ergeben. So konnte beispielsweise die SAMPRISS-Studie zeigen, dass die Behandlung von intrakraniellen Stenosen mittels Stent-Angioplastie einer aggressiven medikamentösen Therapie (bestehend aus Lebensstilmodifikation, Blutdrucksenkung, aggressiver Lipid-Therapie sowie Doppelplättchenhemmung) massiv unterlegen ist und $\mathrm{zu}$ mehr Todesfällen und Schlaganfällen führt. Eine interventionelle Versorgung von intrakraniellen Stenosen sollte deshalb nur noch in Ausnahmefällen durchgeführt werden. Im Bereich der Behandlung von Patienten mit Vorhofflimmern stellen die sogenannten direkten oralen Antikoagulantien eine neue Therapiealternative dar, welche im Vergleich zu den Vitamin-K-Antagonisten ein niedrigeres Hirnblutungsrisiko aufweisen. Weitere Studien laufen zu der Frage, wie bereits auf der Stroke Unit bestmöglich ein Vorhofflimmern nachgewiesen werden kann und wie sowohl diagnostisch als auch therapeutisch bei Patienten mit kryptogenen Hirninfarkten verfahren werden soll. Kardiologische Interventionen bieten mögliche Therapiealternativen: So gibt es erste Studien zum Einsatz interventioneller Herzohrverschlüsse zur Schlaganfall-Prävention bei Vorhofflimmern. Ob die Katheterablation langfristig Schlaganfälle reduzieren kann, ist bislang völlig unklar; derzeit sollten alle Patienten mit entsprechendem Schlaganfall-Risiko auch nach einer solchen Intervention langfristig antikoaguliert werden. Auch Lebensstilinterventionen gehören zu einer effektiven Schlaganfall-Prävention: dies betrifft die regelmäßige körperliche Aktivität, gesunde salzarme Ernährung, moderaten Alkoholgenuss sowie psychosoziale Faktoren. Zusätzlich zur klinischen Evidenz sind gerade zu diesen Themen in den letzten Jahren eine Anzahl experimenteller Untersuchungen durchgeführt worden, welche die zugrundeliegenden Mechanismen darlegen können. 
Forschungsförderung: DFG (Exzellenzcluster NeuroCure; SFB TR 43, KFO 247, KFO 213), BMBF (Centrum für Schlaganfallforschung Berlin), EU (Eustroke, ARISE, WakeUp), Volkswagen Stiftung (Lichtenberg Programm), Corona Stiftung

Interessenkonflikt: Forschungsförderung: AstraZeneca, Sanofi, Roche

Beratungstätigkeit: Bayer, Boehringer Ingelheim, BristolMyers Squibb, MSD, Pfizer, Sanofi

Vortragshonorare: AstraZeneca, Bayer, Boston Scientific, Berlin Chemie, Bristol-Myers Squibb, Boehringer Ingelheim, De- sitin, Edwards, Ever, GlaxoSmithKline, MSD, Novartis, Pfizer, Sanofi, Servier, Takeda, Trommsdorff.

Bibliografie

DOI http://dx.doi.org/10.1055/s-0033-1346712

Drug Res 2013; 63, Suppl. 1: S14-S15

(c) Georg Thieme Verlag KG Stuttgart · New York .

ISSN 2194-9379 